\title{
Genome-wide association study identifies two risk loci for tuberculosis in Han Chinese
}

Ruijuan Zheng ${ }^{1}$, Zhiqiang $\mathrm{Li}^{2,3}$, Fusheng $\mathrm{He}^{4}$, Haipeng Liu (1) ${ }^{1}$, Jianhua Chen ${ }^{3}$, Jiayu Chen ${ }^{5}$, Xuefeng Xie ${ }^{4}$, Juan Zhou ${ }^{3}$, Hao Chen ${ }^{6}$, Xiangyang Wu ${ }^{6}$, Juehui Wu ${ }^{6}$, Boyu Chen ${ }^{3}$, Yahui Liu ${ }^{3}$, Haiyan Cui ${ }^{7}$, Lin Fan ${ }^{7}$, Wei Sha ${ }^{7}$, Yin Liu ${ }^{8}$, Jiqiang Wang ${ }^{3}$, Xiaochen Huang ${ }^{1}$, Linfeng Zhang ${ }^{5}$, Feifan $X_{u^{9}}{ }^{\text {, Jie Wang}}{ }^{1}$, Yonghong Feng ${ }^{1}$, Lianhua Qin', Hua Yang ${ }^{1}$, Zhonghua Liu ${ }^{1}$, Zhenglin Cui ${ }^{1}$, Feng Liu ${ }^{1}$, Xinchun Chen ${ }^{10}$, Shaorong Gao ${ }^{5}{ }^{5}$,

Silong Sun ${ }^{4}$, Yongyong Shi (id ${ }^{2,3,11} \&$ Baoxue Ge $e^{1,6}$

Tuberculosis (TB) is an infectious disease caused by Mycobacterium tuberculosis (Mtb), and remains a leading public health problem. Previous studies have identified host genetic factors that contribute to Mtb infection outcomes. However, much of the heritability in TB remains unaccounted for and additional susceptibility loci most likely exist. We perform a multistage genome-wide association study on 2949 pulmonary TB patients and 5090 healthy controls (833 cases and 1220 controls were genome-wide genotyped) from Han Chinese population. We discover two risk loci: $14 \mathrm{q} 24.3\left(\mathrm{rs} 12437118, P_{\text {combined }}=1.72 \times 10^{-11}, \mathrm{OR}=1.277, \mathrm{ESRRB}\right)$ and $20 \mathrm{p} 13$ (rs6114027, $\left.P_{\text {combined }}=2.37 \times 10^{-11}, \mathrm{OR}=1.339, \mathrm{TGM6}\right)$. Moreover, we determine that the rs6114027 risk allele is related to decreased TGM6 transcripts in PBMCs from pulmonary TB patients and severer pulmonary TB disease. Furthermore, we find that tgm6deficient mice are more susceptible to Mtb infection. Our results provide new insights into the genetic etiology of TB.

\footnotetext{
${ }^{1}$ Shanghai Key Lab of Tuberculosis, Shanghai Pulmonary Hospital, Tongji University School of Medicine, Shanghai 200043, China. ${ }^{2}$ The Affiliated Hospital of Qingdao University \&The Biomedical Sciences Institute of Qingdao University (Qingdao Branch of SJTU Bio-X Institutes), Qingdao University, Qingdao 266003, China. ${ }^{3}$ Bio-X Institutes, Key Laboratory for the Genetics of Developmental and Neuropsychiatric Disorders (Ministry of Education) and the Collaborative Innovation Center for Brain Science, Shanghai Jiao Tong University, Shanghai 200030, China. ${ }^{4}$ BGI Genomics, BGI-Shenzhen, Shenzhen 518083, China. ${ }^{5}$ Clinical and Translational Research Center of Shanghai First Maternity and Infant Hospital, Shanghai Key Laboratory of Signaling and Disease Research, School of Life Science and Technology, Tongji University, Shanghai 200092, China. ${ }^{6}$ Department of Microbiology and Immunology, Tongji University School of Medicine, Shanghai 200092, China. ${ }^{7}$ Tuberculosis Center for Diagnosis and Treatment, Shanghai Pulmonary Hospital, Tongji University School of Medicine, Shanghai 200043, China. ${ }^{8}$ Department of Clinical Laboratory, Shanghai Pulmonary Hospital, Tongji University School of Medicine, Shanghai 200043, China. ${ }^{9}$ Clinical Laboratory, The Sixth People's Hospital of Nantong, Jiangsu 226011, China. ${ }^{10}$ Shenzhen University School of Medicine, Shenzhen 518060, China. ${ }^{11}$ Department of Psychiatry, First Teaching Hospital of Xinjiang Medical University, Urumqi 830054, China. These authors contributed equally: Ruijuan Zheng, Zhiqiang Li, Fusheng He. Correspondence and requests for materials should be addressed to H.L. (email: haipengliu@tongji.edu.cn) or to S.S. (email: sunsilong@genomics.cn) or to Y.S. (email: shiyongyong@gmail.com) or to B.G. (email: gebaoxue@sibs.ac.cn)
} 
$\mathrm{T}$ uberculosis (TB), an infectious disease caused by $M y c o-$ bacterium tuberculosis $(M t b)$, remains the leading cause of death by infection in the world ${ }^{1}$, with 10.4 million new cases and 1.7 million deaths in $2016^{2}$. Substantial evidence from twin $^{3,4}$, family linkage, and candidate gene studies ${ }^{6}$ has demonstrated that host genetic factors are key players in determining the susceptibility to TB.

Four common variants associated with TB have been identified through genome-wide association studies (GWAS) in African and/or Russian populations ${ }^{7-9}$. However, some of these variants have much lower frequencies in other populations, making the data difficult to replicate ${ }^{10-13}$. Besides, the human leukocyte antigens (HLA) class II region was found to contribute to genetic risk of TB in Europeans ${ }^{14}$. Another GWAS performed in an Asian population did not identify susceptibility loci that reached the threshold for genome-wide significance ${ }^{15,16}$, likely because of smaller sample sizes. As much of the heritability in TB remains unaccounted for and more susceptibility loci for TB likely remain to be identified, it is valuable to perform GWAS for TB among different ethnic groups to further understanding the genetic basis of TB.

To identify the loci for susceptibility to TB, we perform a threestage GWAS in the Han Chinese population using 2949 pulmonary TB cases and 5090 controls. We identify two susceptibility loci that are significantly associated with TB at $14 \mathrm{q} 24.3$ (rs12437118, $P=1.72 \times 10^{-11}$ for ESRRB) and 20p13 (rs6114027, $P=2.37 \times 10^{-11}$ for TGM6). Moreover, we find the rs6114027 regulates the expression of the TGM6 gene and is associated with the severity of TB. Furthermore, by generating tgm6-deficient mice we demonstrate that tgm 6 protects mice from $M t b$ infection. Taking together, our findings provide shed light on the genetic etiology and pathogenesis of TB.

\section{Results}

GWAS and replications. To identify susceptibility loci for TB, we performed a three-stage GWAS of TB in a Han Chinese population. We conducted genome-wide genotyping of 2112 subjects using Affymetrix Axiom CHB arrays (discovery stage). After quality control (QC) and imputation, a total of $5,374,021$ variants with a minor allele frequency (MAF) of $3 \%$ or greater in 833 pulmonary tuberculosis cases and 1220 controls were analyzed (Supplementary Table 1). We utilized principal component analysis (PCA) to detect potential population stratification in our samples (Supplementary Figure 1) and conducted logistical regression analysis adjusted for the first three principal components in the discovery stage. After PCA-adjustment, we did not observe significant inflation of test statistics (inflation factor $\lambda=1.01$, Supplementary Figures 2 and 3 ), suggesting that effects from potential population stratification were well-controlled.

In the discovery stage, none of the single nucleotide polymorphisms (SNPs) achieved the genome-wide significance threshold $\left(P\right.$ value $\left.=5 \times 10^{-8}\right)$ for the Affymetrix Axiom ${ }^{\oplus}$
Genome-Wide CHB1 and CHB2 Array Plate Set (two chips/ set). We genotyped the lead SNPs or their proxies from the top ten most significantly associated independent loci $\left(P<1 \times 10^{-5}\right.$, Supplementary Data 1) in two independent sample sets. Replication sample set 1 was comprised of 1074 cases and 1904 controls; replication sample set 2 was comprised of 1042 cases and 1966 controls (Supplementary Table 1). The combined analysis of the discovery and replication stages gave a total sample size of 2949 cases and 5090 controls, which can provide sufficient statistical power $(>85 \%)$ to detect associations with genotypic relative risks (GRR) of 1.30 for variants with risk-allele frequencies (RAF) of $\sim 20$ to $75 \%$ (Supplementary Table 2). In the replication stage, six SNPs had signals with the same direction of effect as was observed in the discovery analysis, and three of them showed significance $(P<0.05)$ (Supplementary Table 3$)$. In the combined analysis two SNPs (rs12437118 at 14q24.3 and rs6114027 at 20p13) were genome-wide significantly associated with TB. Both SNPs showed significance $(P<0.05)$ in all the three data sets and were consistent across data sets (Table 1). We also queried epigenetic annotations for 14q24.3 and 20p13 (indexed by rs12437118 and rs6114027, respectively) to explore potential implications of the association signals. The index SNPs and most of their linked variants $\left(r^{2}>0.2\right)$ are predicted to have putative regulatory function (Supplementary Data 2).

The most significant signal was observed at rs12437118 $\left(P_{\text {combined }}=1.72 \times 10^{-11}\right.$, odds ratio $(\mathrm{OR})=1.277$, Fig. $\left.1 \mathrm{a}\right)$ at 14q24.3, the allele frequencies for risk allele A ranged from 0.261 to 0.288 in the three stages, and no heterogeneity across the stages was found $\left(P_{\text {het }}=0.719\right.$ and heterogeneity index $\left.I^{2}=0\right)$. SNP rs12437118 is located in an intergenic region $16 \mathrm{~kb}$ downstream of ESRRB. Common variations within this region have been associated with autosomal-recessive nonsyndromic hearing impairment ${ }^{17}$, acute lymphoblastic leukemia (childhood) ${ }^{18}$, and healing of the rotary cuff $^{19}$.

The second significant signal was observed at rs6114027 $\left(P_{\text {combined }}=2.37 \times 10^{-11}, \mathrm{OR}=1.339\right.$, Fig. $\left.1 \mathrm{~b}\right)$ at $20 \mathrm{p} 13$, the allele frequencies for risk allele $\mathrm{C}$ ranged from 0.153 to 0.160 in the stages, and no heterogeneity was observed $\left(P_{\text {het }}=0.399\right.$ and $I^{2}=$ $0)$. SNP rs6114027 is located in the intron region of the TGM6 gene. The protein encoded by TGM6 belongs to the transglutaminase superfamily that catalyzes cross-linking of proteins and conjugation of polyamines to proteins. Mutations in this gene have been reported to be associated with spinocerebellar ataxia type 35 (SCA35) $^{20}$ and acute myeloid leukemia ${ }^{21}$.

We investigated the association of the SNPs identified in the published TB GWAS ${ }^{7-9,14}$ with susceptibility to TB based on our data set. Four SNPs located outside the HLA region were highlighted in previous reports ${ }^{7-9}:$ rs4733781 and rs10956514 in the ASAP1 gene and rs 2057178 on chromosome 11 p13 as well as rs4331426 on chromosome 18q11.2. For all these SNPs the direction of the effects analyzed in our GWAS were consistent with published findings. For rs2057178 and rs4331426, the OR values are comparable for our discovery set as well as previous

Table 1 Genome-wide significant association of two independent SNPs with TB

\begin{tabular}{|c|c|c|c|c|}
\hline Chr14:76983730 & Replication 1 & $A(0.269)$ & $1.292[1.149-1.452]$ & $1.72 \mathrm{E}-05$ \\
\hline ESRRB & Combined & & $1.277[1.189-1.371]$ & $1.72 \mathrm{E}-11$ \\
\hline rs6114027 & Discovery & $C(0.153)$ & $1.480[1.244-1.760]$ & $9.37 \mathrm{E}-06$ \\
\hline Chr20:2379598 & Replication 1 & $C(0.160)$ & $1.285[1.116-1.479]$ & $4.92 \mathrm{E}-04$ \\
\hline TGM6 & Replication 2 & $C(0.158)$ & $1.306[1.138-1.499]$ & $1.45 \mathrm{E}-04$ \\
\hline
\end{tabular}



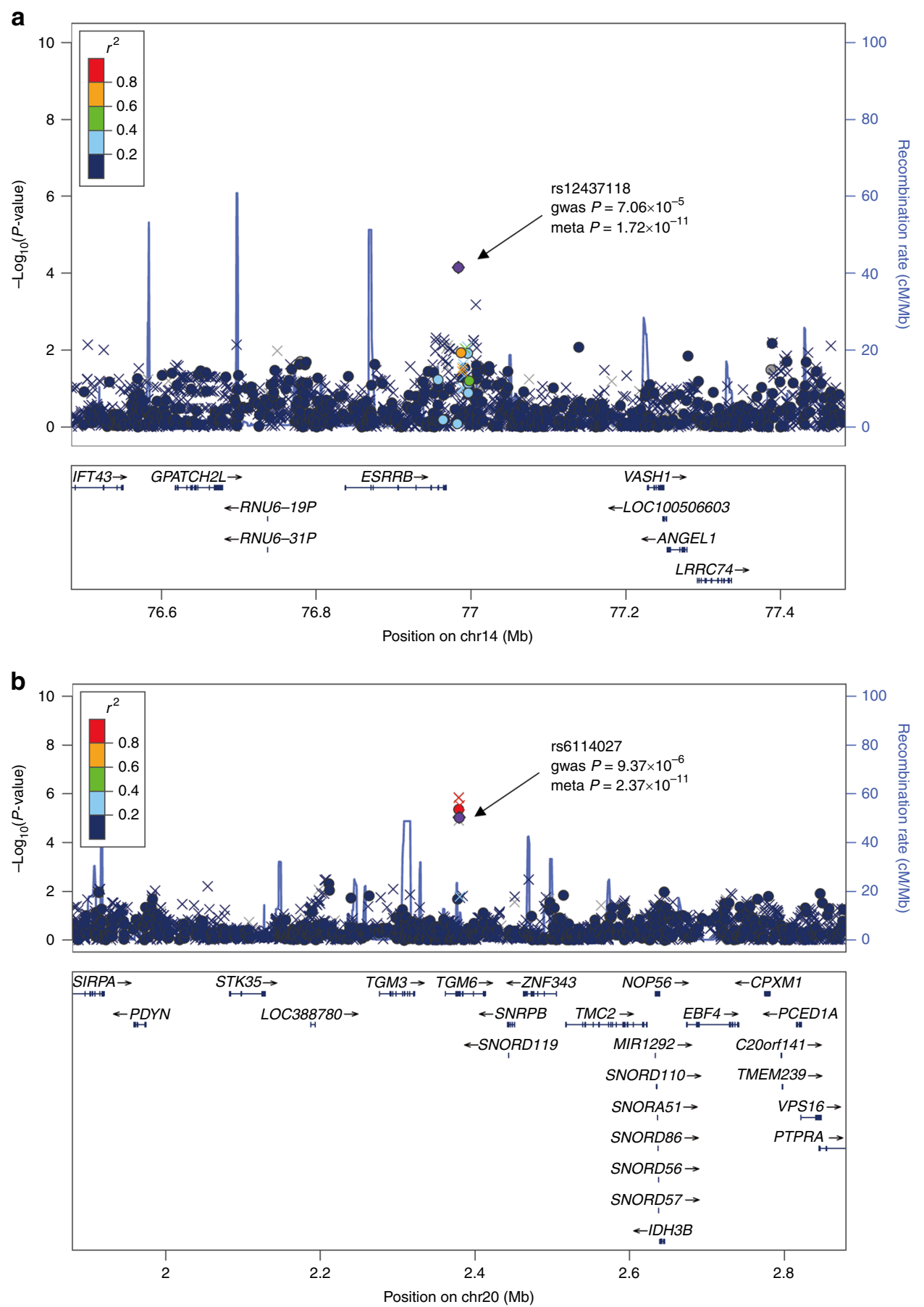

Fig. 1 Regional association plots of loci associated with TB. a rs12437118 and $\mathbf{b}$ rs6114027. Purple circles represent the marker SNP in each region in the meta-analysis of discovery and replication. $-\log _{10} P$ values ( $y$ axis) of the SNPs (within the regions spanning $500 \mathrm{~kb}$ on either side of the marker SNP) are presented according to the chromosomal positions of the SNPs ( $x$ axis, hg19). Genotyped and imputed SNPs are shown with circle and cross symbols, respectively. SNPs are shown in different colors based on their linkage disequilibrium (LD) with the marker SNP, which were established based on the 1000 Genome Asian (ASI) data (March 2012). Recombination rates estimated from the 1000 Genomes Project March 2012 samples are shown as blue lines, and within the interested regions, the genomic locations of genes annotated from the UCSC Genome Browser are displayed as arrows

studies ( 0.88 vs. 0.82 and 1.19 vs. 1.19 , respectively). However, none of the SNPs achieved the genome-wide significance threshold in our data set (Supplementary Table 4), which might be due to the rarity $(<5 \%)$ of these SNPs in Chinese populations as well as the limited sample size of our discovery data set. Three SNPs (rs557011, rs9271378, and rs9272785) in the HLA region were found to be genome-wide significantly associated with TB phenotypes in Europeans ${ }^{14}$. We observed nominal significant association with TB risk in Chinese samples for rs557011 and rs9271378 (located between HLA-DQA1 and $H L A-D R B 1, P<0.05$ and with a consistent direction of effect, Supplementary Table 4), but failed to replicate the association for rs9272785 (located in $H L A-D Q A 1)$. We also imputed HLA alleles of $H L A-A, H L A-B$, HLA-C, HLA-DPA1, HLA-DPB1, HLA-DQA1, HLA-DQB1, and HLA-DRB1 using SNP2HLA ${ }^{22}$ with a Han Chinese reference panel $^{23}$. Like rs9272785, our data did not support the 

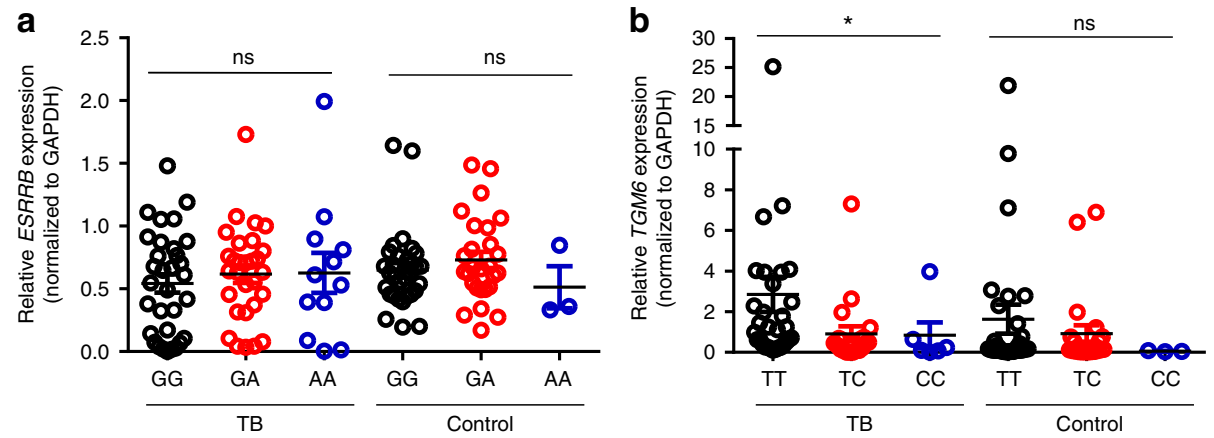

Fig. 2 Association of SNPs with mRNA expression in PBMCs. Genomic DNA and mRNA were isolated from PBMCs from 73 TB patients (TB) and 61 healthy individuals (Control). ESRRB and TGM6 mRNA expression was measured via quantitative RT-PCR and normalized to GAPDH. a The association between ESRRB mRNA expression and rs12437118 genotype. b The association between TGM6 mRNA expression and rs6114027 genotype. Each symbol represents an individual. Data are representative of one independent experiments. Data shown were mean \pm SD and statistical significance was determined via linear regression, $* P<0.05$; ns, not significant

HLA-DQA ${ }^{*}$ 03:01 (the most significantly associated HLA allele in Europeans) ${ }^{14}$ as a risk factor for TB in Chinese (Supplementary Data 3 ). None genome-wide significant association was observed for all the tested HLA alleles (Supplementary Data 3).

We further conducted complementary gene and pathwaybased association analyses for leveraging our genome-wide data set. None of the tested genes or pathways reached significance after Bonferroni correction $\left(P<2 \times 10^{-6}\right)$. In the gene-based analysis, the most associated gene was $B M P 3\left(P=9.53 \times 10^{-5}\right.$, Supplementary Data 4). In addition, two other biologically plausible genes for TB (IL18R1 and IL1RL1) were also found in the top ranked gene list $\left(P=5.24 \times 10^{-4}\right.$ and $6.77 \times 10^{-4}$, respectively). Of the top five most significant gene sets in our pathway-based analysis, four are adhesion related $(P=4.83 \times 10$ ${ }^{-5}$ to $6.79 \times 10^{-6}$, Supplementary Data 5 ).

Functional assessments. To explore the potential biological function of rs12437118 and rs6114027, the expression quantitative trait locus (eQTL) mapping was conducted in peripheral blood mononuclear cells (PBMCs) of $73 \mathrm{~TB}$ patients and 61 healthy individuals. The results showed that the rs12437118 was not associated with ESRRB mRNA expression in PBMCs (Fig. 2a). For rs6114027, C allele carriers with a higher risk for TB had significantly lower TGM6 expression in PBMCs than non- $\mathrm{C}$ allele carriers harboring a lower TB risk (Fig. 2b). SNP rs6114027 is located in the intron between exon 5 and 6 of the TGM6 gene (Supplementary Figure $4 \mathrm{a}$ ). We then interrogated whether the intron region including the allele affects TGM6 gene expression by function as an enhancer or suppressor. To this end, we generated a promoter reporter construct containing a $1514 \mathrm{bp}$ intron fragment with the SNP rs6114027 T or C allele (Supplementary Figure $4 \mathrm{~b}$ ) and performed a dual-luciferase assay. We found that the C allele inhibited luciferase expression in HEK293T cells to a significantly greater extent as compared with the $\mathrm{T}$ allele, suggesting this SNP may affect TGM6 expression by modulating the suppressive effect of the intron on gene transcription (Supplementary Figure 4c).

We further analyzed the association of polymorphism of rs6114027 with clinical indices including the plasma levels of cytokines (IL-6, sIL-2R, and TNF- $\alpha$ ), erythrocyte sedimentation rate (ESR) and the presence of cavity in the lung as well as the presence of mycobacteria in the sputum smear (Supplementary Figure 5a). The abundance of plasma IL-6 was significantly lower in the patients with rs6114027CC genotype than those with rs6114027TT genotype (Supplementary Figure 5b). Moreover, TB patients carrying rs6114027CC genotype showed significantly higher level of plasma sIL-2R than those with rs6114027TT genotype (Supplementary Figure 5c). However, the abundance of plasma TNF- $\alpha$ and ESR were comparable among TB patients carrying different rs6114027 genotypes (TT, TC, and CC) (Supplementary Figure 5d, e). Of note, TB patients carrying rs6114027CC genotype showed significantly higher frequencies of mycobacteria in the sputum smear, but no difference in the lung cavity, than those with rs6114027TT genotype (Supplementary Figure 5f, g), indicating that the rs6114027C allele is a predisposing factor for severer pulmonary TB disease.

To assess the role of TGM6 in the pathogenesis of TB in vivo, we generated tgm6-deficient mice by CRISPR/Cas9-mediated genome editing 24,25 (Supplementary Figure 6). We found that tgm6 homozygous knockout mice are not lethal (Supplementary Figure 7a). There are no apparent differences in the gross phenotype such as body weight among the wild type, tgm6 heterozygous and homozygous mice (Supplementary Figure 7a, b). All three genotypes of mice were employed for $M t b$ infection intraperitoneally. Both histopathological impairments and bacterial burden in the lung were examined. The result demonstrated that tgm6-deficient mice had the most robust infiltration of immune cells and inflammatory lesions among all three genotypes, indicating that $M t b$ infection led to severest tissue damage in the lung of tgm6-deficient mice (Fig. 3a, b). The bacterial burdens in the lung tissue as detected by both acid-fast staining and CFU assay were higher in tgm6-deficient mice than those in either heterozygous or wild type mice (Fig. 3c, d). Furthermore, we measured the expression of cytokines and chemokines in the lung of infected mice and found tgm6-deficient mice harbored the highest levels of $i l-10, \operatorname{tnf}-\alpha$, and $c c l-3$ mRNA among all three genotypes (Fig. 3e). Taken together, $\operatorname{tgm} 6$ confers protection of mice against $M t b$ infection likely in dose-dependent manner.

\section{Discussion}

In this three-stage GWAS of TB, we identified two loci on $14 \mathrm{q} 24.3$ derived from ESRRB and on 20p13 derived from TGM6 that were significantly associated with TB risk in a Chinese Han population. We further demonstrated that the rs6114027C allele was associated with lower expression levels of TGM6 in the PBMCs of TB patients, and tgm6 deficiency rendered increased susceptibility to $M t b$ infection in mice.

The top signal SNP (rs12437118) is localized in an intergenic region $16 \mathrm{~kb}$ downstream of the estrogen-related receptor beta $(E S R R B)$ gene. ESRRB is a member of the orphan nuclear receptor subgroup of the nuclear receptor superfamily ${ }^{26}$. It has been reported that the ESRRB gene is expressed in all human tissues, including the lung ${ }^{17}$. ESRRB plays a critical role in the 
a

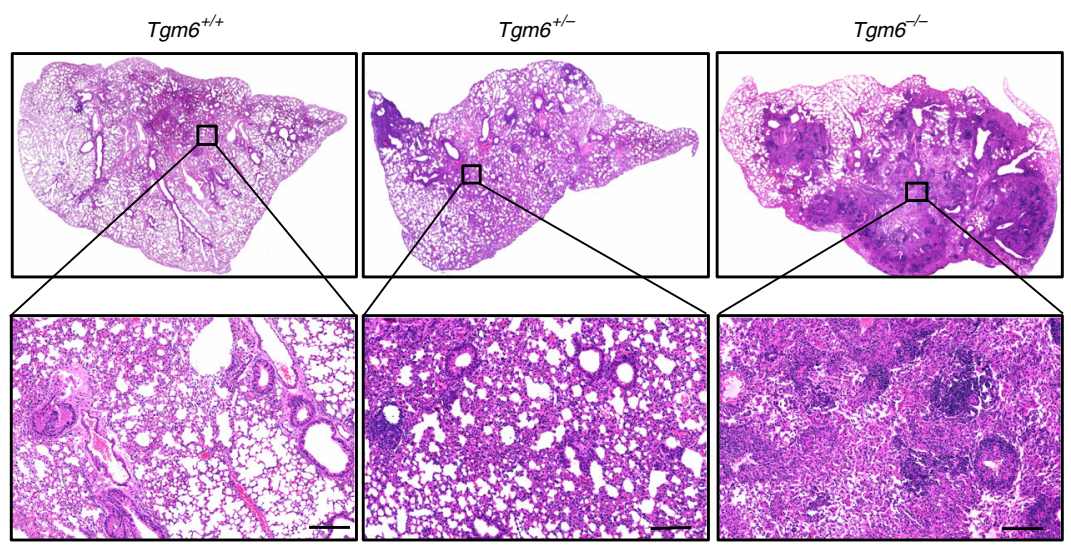

C
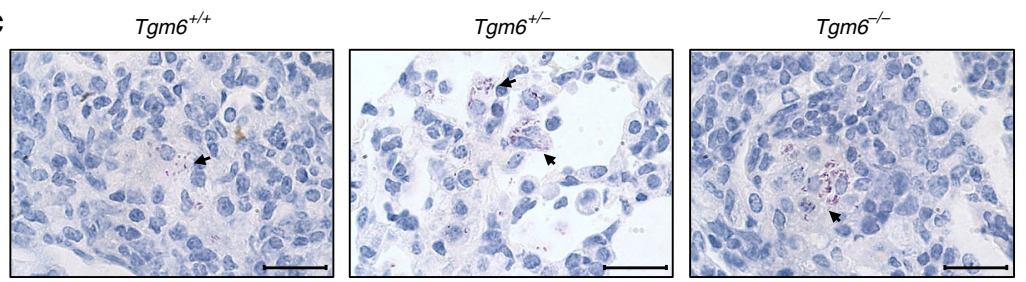

b

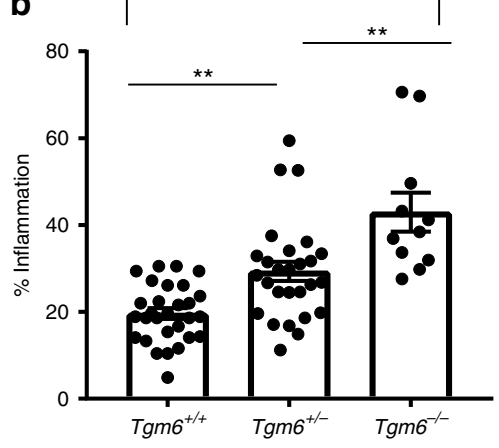

d

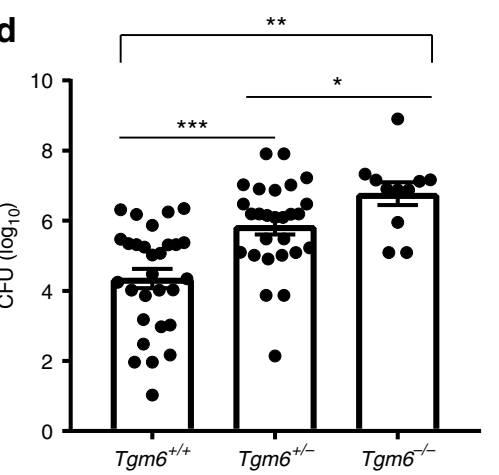

e

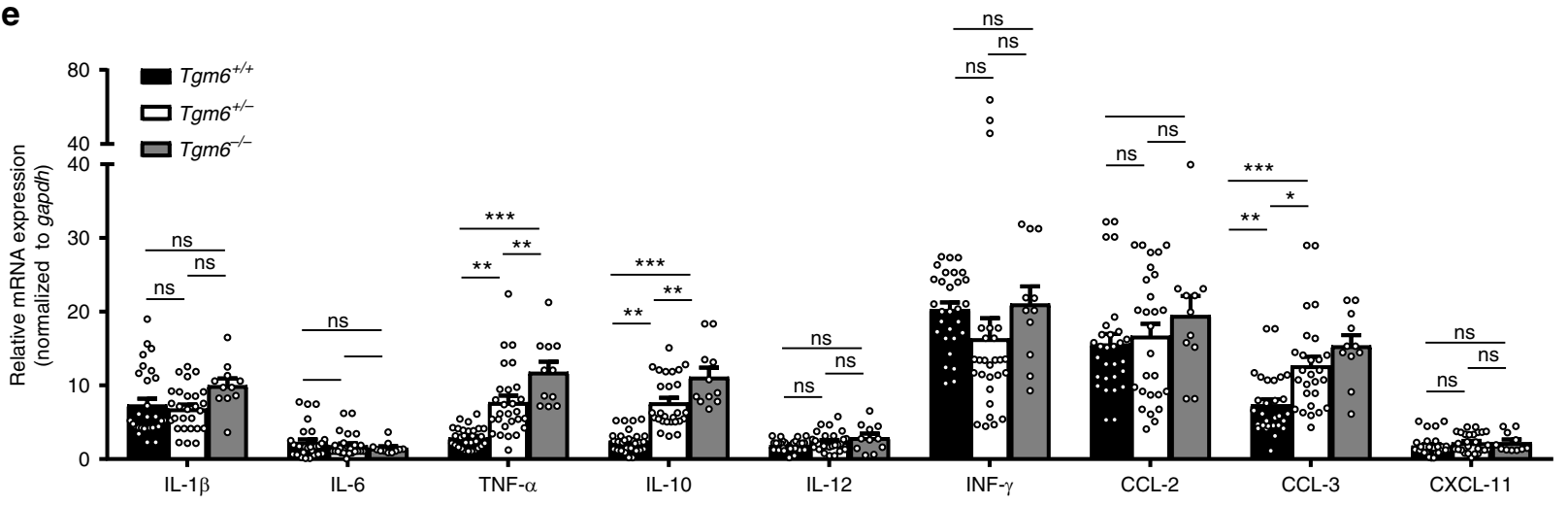

Fig. $3 \mathrm{Tgm6}^{-/-}$mice are more susceptible to Mtb infection. a H\&E staining of the lungs from Mtb-infected mice of indicated genotype. The upper row showing the representative picture of the whole lobe of the stained lung from mice. The lower row showing the enlarged vision of the selected region. Scale bar, $200 \mu \mathrm{m}$; magnification: $\times 100$. b Results showing the quantification of the percentages of inflammation regions as measured by H\&E staining in one lobe of the lung from mice of indicated genotype. Data shown are mean \pm SEM. One-way ANOVA followed by the Bonferroni post hoc test were used for statistical analyses. c Representative results of acid-fast staining of Mtb in the lungs from mice infected with Mtb. Scale bar, $20 \mu$ m; magnification: $\times 1000$. d The bacterial burden in the lungs from Mtb-infected mice was measured by a CFU assay. Data were presented as the medians \pm interquartile ranges and were analyzed by the Mann-Whitney $U$ test. e Quantitative RT-PCR measurement of the mRNA levels (normalized to gapdh) of cytokines and chemokines in lungs from infected mice of indicated genotypes. The data for $\operatorname{tgm} 6^{+/+}(n=29)$ and $\operatorname{tgm} 6^{+/-}(n=27)$ mice were pooled from four independent experiments and the data for $\operatorname{tgm} 6^{-1-}$ mice $(n=11)$ were pooled from two independent experiments. Data shown were mean \pm SEM. One-way ANOVA followed by the Bonferroni post hoc test were used for statistical analyses. ${ }^{*} P<0.05$; ${ }^{* *} P<0.01 ; * * * P<0.001$; ns, not significant

development of several tissues and organ systems and esrrb knockout mice die at 10.5 days postcoitum ${ }^{27}$. ESRRB is involved in the development of the inner ear and hearing, ${ }^{28}$ conditional esrrb ${ }^{-1-}$ mice show a defect in vestibular function and hearing impairment. In humans, mutations in the $E S R R B$ gene were found in autosomal-recessive nonsyndromic hearing impairment DFNB35 29,30 . Until now, the function of ESRRB in TB infection was unknown. A previous study indicated that ESRRB directly bound to Gata6 promoter and resulted in maintenance of the undifferentiated status of embryonic stem cells ${ }^{31}$. Interestingly, GATA6 was previously thought to be a TB susceptible gene due to close proximity to a susceptibility locus for $\mathrm{TB}$ on chromosome
$18 \mathrm{q} 11.2$ variant (SNP rs4331426) identified by GWAS ${ }^{7}$. GATA6 is required for both lung development and regeneration by balancing stem/progenitor expansion and epithelial differentiation via Wnt signaling ${ }^{32}$. Our GWAS analyses determined that the ESRRB gene was significantly associated with TB. This make it tempting to hypothesize that ESRRB contributes to TB pathogenesis by regulating the regeneration of damaged lung tissue caused by $M t b$ infection, which warrants additional studies. However, no difference was detected between rs12437118 and ESRRB mRNA in PBMCs. It is, therefore, highly likely that the differences in ESRRB abundances across the different genotypes may be restricted to some other tissues. 
The second identified SNP, rs6114027, was mapped to the intron region of the TGM6 gene. The evidence suggests that TGM6 plays an important role in neurologic diseases; however, the exact mechanism of action remains elusive. Via exome sequencing and family linkage analysis, TGM6 has been identified as a causative gene of spinocerebellar ataxias ${ }^{20}$, and acute myeloid leukemia $^{21}$. Several identified mutations of TGM6 gene were reported to be prone to cause death of primary neurons resulting from unfolded protein reaction activation, which may contribute to pathogenesis of SCA $35^{33}$. As an autoimmune target, antiTGM6 antibodies were presented at a higher level in patients with progressive multiple sclerosis $(\mathrm{MS})^{34}$, amyotrophic lateral sclerosis $^{35}$, and gluten-sensitive cerebellar ataxia ${ }^{36,37}$. In this study, we identify a mutation in TGM6 associated with TB; our eQTL analysis showed that the rs6114027C allele was associated with significantly lower expression levels of TGM6 in PBMCs from TB patients. The luciferase assay further validated a suppressive effect of SNP rs6114027C allele on gene transcription. Most risk alleles have been found to alter transcription-regulatory element binding ${ }^{38}$, however, whether this intronic SNP regulates gene expression via modulating suppressor activity warrants further investigation.

Here, we report an important role of TGM6 in the process of $M t b$ infection. Aerosol route using inhalation exposure system is more physiologic and relevant to natural infection, which is more favored for $M t b$ infection in mouse model. However, due to the inaccessibility to the aerosol infection model, we employed an intraperitoneal injection model, which has been reported previously ${ }^{39-41}$. We successfully generated a strain of tgm6 knockout mice with a protein null mutant by CRISPR/Cas9-mediated genome editing. No gross phenotypic defects were observed in both tgm 6 heterozygous and homozygous mice. However, tgm6 deficiency led to a higher bacterial burden and more pathological impairments in the lung of mice post $M t b$ infection. Moreover, we observed that the deletion of $\operatorname{tgm} 6$ resulted in elevated transcripts of $i l-10, t n f-\alpha$, and $c c l-3$ mRNA in the lung of $M t b$-infected mice. However, whether the enhanced cytokines response is secondary to the increased bacterial burden or directly modulated by TGM6 warrants further investigation.

By analyzing the association of polymorphism of rs6114027 with clinical indices, we found that the rs6114027C allele is associated with severer pulmonary TB disease as manifested with higher frequencies of mycobacteria in the sputum smear. Notably, the elevated TGM6 in astrocytes was found to regulate the expression of glial fibrillary acidic protein (GFAP) in progressive $\mathrm{MS}^{34}$. As a marker of astrocyte activation, GFAP reflects the level of damage to the blood-brain barrier to some extent. Of note, GFAP is highly expressed in the brain of tuberculosis meningitis, a fatal form of $M t b$ infection of the central nervous system ${ }^{42}$. Therefore, how TGM6 regulates the dissemination of $M t b$ and development of severe TB such as extrapulmonary TB remains an interesting question to be addressed.

In the gene-based analysis, the top gene associated with TB was bone morphogenetic protein 3 (BMP3). BMP3 is a member of the transforming growth factor-beta superfamily and regulates osteoblast differentiation. To identify TB-risk associated biological pathways, we performed pathway-based association analysis. Of note, among these identified pathways, the top four pathways were related to adhesion including the adherens junction organization pathway, the focal adhesion assembly pathway, the cell substrate adherens junction assembly pathway as well as the adherens junction assembly pathway. These pathways were important for the regulation of hematopoietic progenitor cells and were essential for both immune and inflammatory responses. It has been reported mycobacterium infection decreased $\beta$-catenin expression, which is an adherens junction protein that promotes cell-cell adhesion and contributes to the control of pleural permeability, leading to TB pleurisy ${ }^{43}$.

The newly identified TB associated SNPs in Chinese (rs12437118 and rs6114027) also are common (MAF: 0.10-0.35, Supplementary Table 5) in African and European populations, but our findings were not seen previously ${ }^{7-9,14}$. Such discrepancy in GWAS findings on the susceptibility loci to TB across populations have been encountered previously. The ASAP1 locus identified in Russian ${ }^{9}$ is failed to be replicated in Icelandic ${ }^{14}$, and Chinese individuals. The inconsistence in GWAS may be due to different causal variants or linkage disequilibrium structures in different populations, or phenotypic heterogeneity. Of note, as a complex infectious disease, TB is an outcome of the intricate interaction between genome and environment. The putative environmental influences may result in genome-wide epigenetic modification, which in turn regulates the pathways relevant to TB pathogenesis in cooperation with genetic variation and gene expression $^{44}$. The validation of a protective effect of TGM6 in the process of $M t b$ infection in mice indicates the reliability of our GWAS study in Han Chinese population.

Taken together, our GWAS in a Chinese population has identified two genetic variants of the ESRRB and TGM6 genes that are associated with susceptibility to TB in Chinese Han population. Our results provide the insight into the genetic etiology of TB and further studies are required to explore how ESRRB and TGM6 are involved in the regulation of $M t b$ infection and in the predisposition to TB.

\section{Methods}

Ethics. All of the participants provided written informed consent. The study was conducted according to the Declaration of Helsinki principles. The protocol was approved by the local ethics committee of Tongji University School of Medicine and signed informed consent was obtained from all subjects (permit number: 2011 FK-03, No. 2015YXY48).

Samples. We performed a three-stage case-control analysis, including an initial discovery stage and two stages of follow-up. TB patients and controls were collected at three Chinese cities: Shanghai (municipality), Nantong (Jiangsu province), and Shenzheng (Guangdong province), according to the same protocol. In the discovery stage, 833 pulmonary tuberculosis patients and 1220 healthy controls recruited from Shanghai Pulmonary Hospital affiliated with Tongji University, were genotyped using an Axiom Genome-Wide CHB1 \& CHB2 Array Plate Set (two chips/set, Affymetrix). During the replication stage, the first-stage validation samples were collected from patients at Shanghai Pulmonary Hospital affiliated with Tongji University (1074 cases and 1904 controls); second-stage validation samples were collected from patients at Shanghai Pulmonary Hospital affiliated with Tongji University (510 cases and 1190 controls), the Sixth People's Hospital of Nantong (297 cases and 398 controls), and Shenzhen Third People's Hospital (235 cases and 378 controls).

Diagnostic criteria for TB were as follows: (1) sputum culture for $M t b$; (2) presence of acid-fast bacilli in sputum smear; (3) clinical presentation and radiological signs (such as X-ray or computed tomography scan). The diagnosis all TB patients was ultimately confirmed by culture of $M t b$ from sputum. Sputum samples were cultured on Löwenstein-Jensen (L.J.) culture media and a BACTEC MGIT 960 system. Identification of the M. tuberculosis complex (MTBC) was performed by the conventional p-nitrobenzoic acid (PNB) and thiophene carboxylic acid hydrazine (TCH) resistance tests. Growth in LJ medium containing PNB indicated that the bacilli did not belong to the MTBC. The patients with extrapulmonary TB were excluded. The flow chart of the sample collection of TB patients was listed in Supplementary Figure 8. The total 3044 culture-positive samples were collected for genomic DNA extraction.

For analysis of association between genetic variation of TGM6 SNP rs6114027 and clinical indices, the clinical data of the samples involved in the discovery stage and the first replication stage were collected, including the plasma levels of cytokines (IL-6, sIL-2R, and TNF- $\alpha$ ), ESR, and the presence of cavity in the lung as well as the presence of mycobacteria in the sputum smear. The sample treated with anti-TB treatment were excluded. The characteristics of the patients were shown in Supplementary Table 6 and the design of the study was in Supplementary Figure 5a.

The controls were recruited from a pool of individuals who participated in a health examination program and were living mainly in the same geographical area as the cases. The control populations were also subject to the physical examination, blood testing, and chest X-rays (CXR) and were screened for a history of TB and a family history of TB; those subjects with signs, symptoms, and CXR results 
suggestive of active TB, a history of previous TB, or anti-TB treatment were excluded. $M t b$ infection status of these controls was unknown. All cases and controls with alcoholism, diabetes, chronic use of corticosteroids, or immunodeficiency were excluded. Controls were not tested for antibodies to human immunodeficiency virus (HIV), but TB cases were tested and excluded if HIV-positive.

Genomic DNA extraction. Venous blood samples were collected with ethylenediaminetetraacetic acid disodium salt (EDTA-2Na)-anticoagulant tubes from all participants, followed by genomic DNA extraction using Flexi Gene DNA kits (Qiagen) and the QuickGene DNA whole-blood kit (Fujifilm) according to the manufacturer's instructions ${ }^{45}$. The extracted DNA was diluted to working concentrations of $50 \mathrm{ng} / \mu \mathrm{l}$ for genome-wide genotyping, and $15-20 \mathrm{ng} / \mu \mathrm{l}$ for the validation study.

Genome-wide genotyping and QC. An Affymetrix Axiom ${ }^{\circledast}$ Genome-Wide CHB1 \& CHB2 Array Plate Set (two chips/set) was used for genome-wide genotyping in the discovery stage. The QC analysis and genotype calling were performed for the CHB1 and CHB2 arrays separately according to the Axiom ${ }^{\circledR}$ Genotyping Solution Data Analysis Guide. Chips with a dish QC (DQC) value less than 0.82 were excluded in the primary QC step. Samples with passing DQC values underwent step 1 genotype calling using a subset of probe sets. Samples with a call rate $<97 \%$ or in a nonpassing plate (the average call rate of passing samples $<98.5 \%$ ) were then excluded. The post-QC samples were kept for Step 2 genotype calling using the Axiom Genotyping Algorithm v1 (Axiom GT1). The SNP polisher was utilized for SNP QC and SNPs in the recommended categories (PolyHighRes, MonoHighRes, and No Minor Homand Hemizygous) were kept, with visual inspection if required or advised.

Next, estimated gender via genotyping data was compared with the sample record, sample with inconsistent gender were removed. Heterozygosity rates were calculated with the intent of removing deviations that exceeded six standard deviations from the mean. PLINK's identity-by-descent analysis was used to detect cryptic relatedness. When a pair of individuals exhibited a PI_HAT $>0.2$, the member of the pair with the lower call rate was excluded from the analysis. SNPs with call rates $<97 \%, \mathrm{MAF}<3 \%$, or significant deviations from the Hardy-Weinberg equilibrium (HWE) in the controls (HWE, $P \leq 1 \times 10^{-6}$ ) were excluded. Ungenotyped SNPs in the GWAS discovery samples were imputed using SHAPEIT $2.0^{46}$ (phasing step), IMPUTE2 ${ }^{47}$ (imputation step) and haplotype information from the 1000 Genomes Project (Phase I integrated variant set across all 1092 individuals, v2, March 2012; see URLs). The variants with INFO $>0.8$ were saved for further analysis, and we also applied the same SNP QC criteria as the aforementioned genotyped analysis. A total of 5,374,021 SNPs passed the quality criteria and were used in the subsequent analyses.

SNP selection and genotyping in replications. We selected independent SNPs with PCA-adjusted and/or unadjusted $P$ values less than $1.0 \times 10^{-5}$ for the replication study. Genotyping for replication was performed using the iPLEX platform (Sequenom, San Diego, CA, Supplementary Data 6). To exclude possible genotyping error, the genotype cluster plots of the selected SNPs for the discovery and replication stages were generated and visually inspected (Supplementary Figures 9 and 10).

Statistical analysis. Population stratification analysis was conducted using a PCAbased method implemented in the software package EIGENSTRAT ${ }^{48}$. Power estimates were calculated for the total sample size used in our study with the GAS Power Calculator ${ }^{49}$, giving a range of GRR and RAF and assuming a population prevalence of $0.004^{50}$, and a significance level of $5 \times 10^{-851}$. Logistic regression was used to test the association of a single SNP using PLINK ${ }^{52}$, and the top three principal components were used as covariates in the association analysis to correct for the population stratification. The independent effects of an individual SNP were evaluated using conditional analysis. In the meta-analysis, we adopted inverse variance weighting fixed-effects model to combine the results from different datasets, and used Cochran's $Q$ test and the $I^{2}$ index to quantify the degree of heterogeneity across the datasets. A Manhattan plot of the $-\log _{10}$ ( $P$ values) was generated using Haploview ${ }^{53}$. Regional plots were generated using the online tool LocusZoom $1.2^{54}$ (see URLs). The Mann-Whitney $U$ test, linear regression and ANOVA followed by Bonferroni post hoc test was performed using Graph-Pad Prism 6.0 software. The genome-wide significant SNPs were annotated using HaploReg ${ }^{55}$ based on the LD information from the Asian 1000 Genomes Project population.

Analysis of association between SNPs and mRNA expression. For validation of the association between genetic variation and ESRRB and TGM6 mRNA expression, $73 \mathrm{~TB}$ patients and 61 healthy individuals were recruited. Two tubes of wholeblood samples were collected from each donor and subjected to DNA extraction and isolation of PBMCs for RNA extraction, respectively.
Peripheral blood mononuclear cells isolation. PBMCs were isolated from EDTA treated whole-blood through density gradient centrifugation by Ficoll separation (Ficoll-Paque plus; Amersham Biosciences) and were then washed with PBS twice. The harvested PBMCs were applied for further experiments.

RT-PCR analysis. TRIzol (Invitrogen) and the ReverTra Ace ${ }^{\circledast P C R}$ RT Kit (Toyobo, FSQ-101) were used for total RNA extraction and the reverse transcription of messenger RNA (mRNA), respectively. The relative mRNA expression of different genes were detected by quantitative real-time RT-PCR with a SYBR RT-PCR kit (Toyobo, QPK-212) and was calculated by comparison with the control gene Gapdh (encoding GAPDH) using the $2^{-} \triangle \triangle \mathrm{Ct}$ method. The primers used are listed in Supplementary Table 7.

Luciferase assay. Human embryonic kidney epithelial cells (HEK293T; ATCC CRL-11268) were maintained in Dulbecco's Modified Eagle Medium (Hyclone) supplemented with $10 \%(\mathrm{v} / \mathrm{v})$ heat-inactivated fetal bovine serum (Gibco) and 100 $\mathrm{U} / \mathrm{ml}$ penicillin and streptomycin. Cell lines were tested for the absence of mycoplasma contamination by PCR. The intron between exon 5 and 6 containing 1514 bp with SNP rs6114027, allele T or C, was cloned into a luciferase reporter vector (pGL3-promoter-TGM6 ${ }^{\mathrm{WT}}$ and pGL3-promoter-TGM6 ${ }^{\mathrm{MT}}$ ). HEK293T cells were plated in 48 -well plates $24 \mathrm{~h}$ before transfection. A total of $0.1 \mu \mathrm{g}$ luciferase reporter vector and $0.1 \mathrm{mg}$ of $\mathrm{pRL}$-TK control vector were co-transfected into the cells using Lipofectamine 2000 reagent (Life technology). At $24 \mathrm{~h}$ post-transfection, cells were harvested and lysed. The Dual-luciferase Reporter Assay System (Promega) was applied for measuring both the firefly and Renilla luciferase activities, and the ratio between firefly and Renilla luciferase activity were calculated to represent promoter activity. The experiments were performed three times with technical duplicates in each independent experiment.

Gene- and pathway-based association analyses. We performed gene and pathway-based association testing using MAGMA ${ }^{56}$. The gene $P$ values were computed using an $F$-test based on a multiple linear regression model and competitive tests were utilized for gene-set analysis. We used the gene sets from the Molecular Signatures Database (MSigDB) databases, which is comprised of eight major collections, including Immune Signatures ${ }^{57}$.

Bacteria. $M t b \mathrm{H} 37 \mathrm{Rv}(\mathrm{H} 37 \mathrm{Rv})$ were grown in Middlebrook 7H9 broth (Becton Dickinson, Cockeysville, MD) with $0.05 \%$ Tween- 80 and $10 \%$ oleic acid-albumindextrose-catalase (OADC) (Becton Dickinson, Sparks, MD).

Mice and Mtb infection. Tgm6 knockout mice was generated by the CRISPR/Cas 9 method $^{24,25}$. The tgm 6 sgRNA were designed by targeting the exon 4 of $\operatorname{tgm} 6$ and the sequences were listed in Supplementary Table 7. C57BL/6n female mice (7-8 weeks old) were used as embryo donors. C57BL/6n female mice were superovulated by intraperitoneally injecting with pregnant mare serum gonadotropin and human choionic gonadotophin, and then mated to $\mathrm{C} 57 \mathrm{BL} / 6 \mathrm{n}$ male mice. The fertilized embryos (zygote) were collected from oviducts and received the injection of mixed Cas 9 mRNA $(100 \mathrm{ng} / \mu \mathrm{L})$ and sgRNA $(25 \mathrm{ng} / \mathrm{ul})$ targeting tgm6, followed by culture in Quinn's Advantage cleavage medium (In-Vitro Fertilization, Inc.) for about $24 \mathrm{~h}$, and every 18-20 two-cell stage embryos were transferred into the oviduct of a pseudo pregnant ICR female mouse at 0.5 days postcoitus $(\mathrm{dpc})$ To determine the nucleotide sequence of mutated alleles, DNA sequencing of F0 mice was performed after TA cloning into plasmid pMD19T (TAKARA). In order to obtain F1 tgm6 knockout mice, F0 mice were crossed with C57BL/6 and newborn generations were genotyped by the Sanger sequencing. Genomic DNA was extracted from tail tips and the PCR-based genotyping were employed. The genotyping primers sequences were listed in Supplementary Table 7. C57BL/6n mice were purchased from Shanghai Laboratory Animal Center (Shanghai, China). All mice were bred in specific pathogen-free conditions at the Laboratory Animal Center of Tongji University. Female mice (7-8 weeks old) were divided randomly into cages upon arrival and were infected by injection of $3.5 \times 10^{5} \mathrm{cfu} \mathrm{Mtb}(\mathrm{H} 37 \mathrm{Rv})$ intraperitoneally (i.p.) in the Biosafety Level-3 (BSL-3) Laboratory for 28 days. The age/sex matched tgm6 wild type littermates were used as controls. All mice experiments were performed in accordance with the University Health Guide for the Care and Use of Laboratory Animals and were approved by the Biological Research Ethics Committee of Tongji University.

Immunoblot analysis. The lung tissues were isolated from mouse and protein was extracted using RIPA Lysis Buffer (Beyotime, China) according to the manufacturer's instructions. The protein sample lysate was separated using $12 \%$ sodium dodecyl sulfate (SDS)-polyacrylamide gel, transferred onto Polyvinylidene Fluoride (PVDF) membrane and incubated with the appropriate antibodies. Rabbi anti-TGM6 (TA331020, 1:1000) was purchased from OriGene Technologies, Inc; rabbit anti-GAPDH (SAB2701826, 1:1000) was purchased from Sigma.

Colony forming unit assay. The infected mice were sacrificed at indicated time points and the lung tissues were harvested and homogenized in $5 \mathrm{ml}$ PBS. Tenfold serial diluents of the lung homogenates were then plated on Middlebrook 7H10 
agar supplemented with 10\% Middlebrook OADC enrichment (both from Becton Dickinson, Sparks, MD) followed by incubation at $37^{\circ} \mathrm{C}$ for around 3 weeks before counting the colonies.

Histological analysis and acid-fast staining. Following fixation with $4 \%$ phosphate-buffered formalin for $24 \mathrm{~h}$, the lung tissues from $M t b$-infected mice were embedded in paraffin wax and were cut into serial sections of 2-3 $\mu \mathrm{m}$ thickness. The infiltration of immune cells into the lungs were detected by Haematoxylin and eosin (H\&E) staining. $M t b$ burden in the lungs were determined by acid-fast staining with standard Ziehl-Neelsen method. Light microscopy was applied for the visualization of the stained slides.

URLs. For PLINK, see http://pngu.mgh.harvard.edu/ purcell/plink/; for EIGENSTRAT, see http://genepath.med.harvard.edu/ reich/Software.htm; for Haploview, see http://www.broadinstitute.org/scientific-community/science/programs/ medical-and-population-genetics/haploview/haploview; for WGAViewer, see http://computel.lsrc.duke.edu/softwares/WGAViewer/; for LocusZoom, see http:// csg.sph.umich.edu/locuszoom/; for META, see http://www.stats.ox.ac.uk/ jsliu/ meta.html; for SHAPEIT, see http://mathgen.stats.ox.ac.uk/genetics_software/ shapeit/shapeit.html; for IMPUTE2, see http://mathgen.stats.ox.ac.uk/impute/ impute_v2.html; for the 1000 Genomes Project, see http://www.1000genomes.org/.

\section{Data availability}

The data that support the findings of this study are available from the corresponding authors on reasonable request. The complete GWAS summary statistics are available at http://analysis.bio-x.cn/gwas/ and https://doi.org/10.6084/m9.figshare.7006310.

Received: 18 July 2017 Accepted: 5 September 2018

Published online: 04 October 2018

\section{References}

1. Dye, C. et al. The population dynamics and control of tuberculosis. Science 328, 856-861 (2010).

2. World Health Organization. WHO Global Tuberculosis Report 2017 (WHO, 2017).

3. Comstock, G. W. Tuberculosis in twins: a re-analysis of the Prophit survey Am. Rev. Respir. Dis. 117, 621-624 (1978).

4. van der Eijk, E. A. et al. Heredity versus environment in tuberculosis in twins: the 1950s United Kingdom Prophit survey: Simonds and Comstock revisited. Am. J. Respir. Crit. Care. Med. 176, 1281-1288 (2007).

5. Grant, A. V. et al. A genome-wide association study of pulmonary tuberculosis in Morocco. Hum. Genet. 135, 299-307 (2016).

6. Khor, C. C. et al. A Mal functional variant is associated with protection against invasive pneumococcal disease, bacteremia, malaria, and tuberculosis. Nat. Genet. 39, 523-528 (2007)

7. Thye, T. et al. Genome-wide association analyses identifies a susceptibility locus for tuberculosis on chromosome 18q11.2. Nat. Genet. 42, 739-741 (2010).

8. Thye, T. et al. Common variants at $11 \mathrm{p} 13$ are associated with susceptibility to tuberculosis. Nat. Genet. 44, 257-259 (2012).

9. Curtis, J. et al. Susceptibility to tuberculosis is associated with variants in the ASAP1 gene encoding a regulator of dendritic cell migration. Nat. Genet. 47, 523-527 (2015).

10. Dai, Y. et al. Fine mapping of genetic polymorphisms of pulmonary tuberculosis within chromosome 18q11.2 in the Chinese population: a case-control study. Bmc. Infect. Dis. 11, 1-6 (2011).

11. Wang, X. et al. Association of polymorphisms in the Chr18q11.2 locus with tuberculosis in Chinese population. Hum. Genet. 132, 691-695 (2013).

12. Ji, L. D. et al. Lack of association between polymorphisms from genome-wide association studies and tuberculosis in the Chinese population. Scand. J. Infect. Dis. 45, 310-314 (2013).

13. Miao, R. et al. Genetic variants at $18 \mathrm{q} 11.2$ and $8 \mathrm{q} 24$ identified by genome-wide association studies were not associated with pulmonary tuberculosis risk in Chinese population. Infect. Genet. Evol. 40, 214-218 (2016).

14. Sveinbjornsson, G. et al. HLA class II sequence variants influence tuberculosis risk in populations of European ancestry. Nat. Genet. 48, 318-322 (2016).

15. Eileen Png et al. A genome wide association study of pulmonary tuberculosis susceptibility in Indonesians. Bmc. Med. Genet. 13, 1-9 (2012).

16. Surakameth Mahasirimongkol et al. Genome-wide association studies of tuberculosis in Asians identify distinct at-risk locus for young tuberculosis. J. Hum. Genet. 57, 363-367 (2012).

17. Collin, R. W. et al. Mutations of ESRRB encoding estrogen-related receptor beta cause autosomal-recessive nonsyndromic hearing impairment DFNB35. Am. J. Hum. Genet. 82, 125-138 (2008).
18. Marincevic-Zuniga, Y. et al. PAX5-ESRRB is a recurrent fusion gene in B-cell precursor pediatric acute lymphoblastic leukemia. Haematologica 101, e20-e23 (2016)

19. Tashiian, R. Z. et al. Identification of a genetic variant associated with rotator cuff repair healing. J. Shoulder Elb. Surg. 25, 865-872 (2016).

20. Guo, Y. C. et al. Spinocerebellar ataxia 35: novel mutations in TGM6 with clinical and genetic characterization. Neurology 83, 1554-1561 (2014).

21. Pan, L. L. et al. Positional cloning and next-generation sequencing identified a TGM6 mutation in a large Chinese pedigree with acute myeloid leukaemia. Eur. J. Hum. Genet. 23, 218-223 (2015).

22. Jia, X. et al. Imputing amino acid polymorphisms in human leukocyte antigenes. PLoS One 8, 1-8 (2013).

23. Zhou, F. et al. Deep sequencing of the MHC region in the Chinese population contributes to studies of complex disease. Nat. Genet. 48, 740-746 (2016).

24. Wang, H. et al. One-step generation of mice carrying mutations in multiple genes by CRISPR/Cas-mediated genome engineering. Cell 153, 910-918 (2013).

25. Liu, W. et al. Dosage effects of ZP2 and ZP3 heterozygous mutations cause human infertility. Hum. Genet. 136, 975-985 (2017)

26. Festuccia, N., Owens, N. \& Navarro, P. Esrrb, an estrogen-related receptor involved in early development, pluripotency, and reprogramming. FEBS Lett. 592, 852-877 (2018).

27. Luo, J. et al. Placental abnormalities in mouse embryos lacking the orphan nuclear receptor ERR-beta. Nature 388, 778-782 (1997).

28. Chen, J. et al. Estrogen-related receptor beta/NR3B2 controls epithelial cell fate and endolymph production by the stria vascularis. Dev. Cell 13, 325-337 (2007).

29. Ben Saïd. et al. A novel missense mutation in the ESRRB gene causes DFNB35 hearing loss in a Tunisian family. Eur. J. Med. Genet. 54, e535-e541 (2011).

30. Collin, R. et al. Mutations of ESRRB encoding estrogen-related receptor beta cause autosomal-recessive nonsyndromic hearing impairment DFNB35. Am. J. Hum. Genet. 82, 125-138 (2008).

31. Uranishi, K. et al. Esrrb directly binds to Gata6 promoter and regulates its expression with Dax1 and Ncoa3. Biochem. Biophys. Res. Commun. 478, 1720-1725 (2016)

32. Yuzhen Zhang. et al. Gene expression profiles and transcriptional regulatory pathways underlying mouse tissue macrophage identity and diversity. Nat. Genet. 40, 862-870 (2008).

33. Tripathy, D. et al. Mutations in TGM6 induce the unfolded protein response in SCA35. Hum. Mol. Genet. 26, 3749-3762 (2017)

34. Cristofanilli, M. et al. Transglutaminase- 6 is an autoantigen in progressive multiple sclerosis and is upregulated in reactive astrocytes. Mult. Scler. 13, 1707-1715 (2017)

35. Gadoth, A. et al. Transglutaminase 6 antibodies in the serum of patients with amyotrophic lateral sclerosis. JAMA Neurol. 72, 676-681 (2015).

36. Hadjivassiliou, M. et al. Autoantibodies in gluten ataxia recognize a novel neuronal transglutaminase. Ann. Neurol. 64, 332-343 (2008).

37. Hadjivassiliou, M. et al. Transglutaminase 6 antibodies in the diagnosis of gluten ataxia. Neurology 80, 1740-1745 (2013).

38. Bernstein, B. E. et al. An integrated encyclopedia of DNA elements in the human genome. Nature 489, 57-74 (2012).

39. Sergey Biketov et al. The role of resuscitation promoting factors in pathogenesis and reactivation of Mycobacterium tuberculosis during intraperitoneal infection in mice. BMC Infect. Dis. 7, 146-146 (2007).

40. Yang, $\mathrm{H}$. et al. Lysine acetylation of DosR regulates the hypoxia response of Mycobacterium tuberculosis. Emerg. Microbes \& Infect. 7, 1-14 (2018).

41. Wang, Y. et al. Long noncoding RNA derived from CD244 signaling epigenetically controls CD8+ T-cell immune responses in tuberculosis infection. Proc. Natl. Acad. Sci. USA 112, E3883-E3892 (2015).

42. Dittrich, S. et al. Blood-brain barrier function and biomarkers of central nervous system injury in rickettsial versus other neurological infections in Laos. Am. J. Trop. Med. Hyg. 93, 232-237 (2015).

43. Mohammed, KA1. et al. Mycobacteria induces pleural mesothelial permeability by down-regulating beta-catenin expression. Lung 181, 57-66 (2003).

44. Hardy, J1 \& Singleton, A. Genome-wide association studies and human disease. N. Engl. J. Med. 360, 1759-1768 (2009).

45. Shi, Y. et al. A genome-wide association study identifies two new cervical cancer susceptibility loci at 4q12 and 17q12. Nat. Genet. 45, 918-922 (2013).

46. Delaneau, O., Zagury, J. F. \& Marchini, J. Improved whole-chromosome phasing for disease and population genetic studies. Nat. Methods 10, 5-6 (2013).

47. Howie, B., Fuchsberger, C., Stephens, M., Marchini, J. \& Abecasis, G. R. Fast and accurate genotype imputation in genome-wide association studies through pre-phasing. Nat. Genet. 44, 955-959 (2012).

48. Price, A. L. et al. Principal components analysis corrects for stratification in genome-wide association studies. Nat. Genet. 38, 904-909 (2006). 
49. Skol, A. D., Scott, L. J., Abecasis, G. R. \& Boehnke, M. Joint analysis is more efficient than replication-based analysis for two-stage genome-wide association studies. Nat. Genet. 38, 209-213 (2006).

50. Wang, L. et al. Tuberculosis prevalence in China, 1990-2010: a longitudinal analysis of national survey data. Lancet 383, 2057-2064 (2014).

51. $\mathrm{Li}, \mathrm{Z}$. et al. Genome-wide association analysis identifies 30 new susceptibility loci for schizophrenia. Nat. Genet. 49, 1576-1583 (2017).

52. Purcell, S. et al. PLINK: a tool set for whole-genome association and population-based linkage analyses. Am. J. Hum. Genet. 81, 559-575 (2007)

53. Barrett, J. C., Fry, B., Maller, J. \& Daly, M. J. Haploview: analysis and visualization of LD and haplotype maps. Bioinformatics 21, 263-265 (2005).

54. Pruim, R. J. et al. LocusZoom: regional visualization of genome-wide association scan results. Bioinformatics 26, 2336-2337 (2010).

55. Ward, L. D. \& Kellis, M. HaploReg: a resource for exploring chromatin states, conservation, and regulatory motif alterations within sets of genetically linked variants. Nucleic Acids Res. 40, D930-D934 (2012).

56. de Leeuw, C. A., Mooij, J. M., Heskes, T. \& Posthuma, D. MAGMA: generalized gene-set analysis of GWAS data. PLoS Comput. Biol. 11, 1-19 (2015).

57. Liberzon, A. et al. Molecular signatures database (MSigDB) 3.0. Bioinformatics 27, 1739-1740 (2011).

\section{Acknowledgments}

We are deeply grateful to all the study participants, research staff, and students who took part in this work. This work was supported by Chinese National Program on Key Basic Research Project (2017YFA0505900), National Natural Science Foundation of China (Nos. 81101217, 81770006, 81370108, 81330069, 91542111, 31730025, 31325014, $81130022,81871055,81701321,81421061,21375139,31571012$, and 81501154), Science and Technology Commission of Shanghai Municipality (No. 16ZR1428800), the National Basic Research Program of China (973 Program) (2015CB559100), the National Key R\&D Program of China (2016YFC0903402, 2016YFC1306903, 2016YFC0902403, and 2017YFC0908105) and the Program of Shanghai Academic Research Leader (15XD1502200), the National Program for Support of Top-Notch Young Professionals, Shanghai Key Laboratory of Psychotic Disorders (13dz2260500), the "Shu Guang" project supported by the Shanghai Municipal Education Commission and Shanghai Education Development Foundation (12SG17), the Shanghai Jiao Tong Univ Liberal Arts and Sciences Cross-Disciplinary Project (13JCRZ02), Shanghai Hospital Development Center (SHDC12016115), Shanghai Mental Health Center (2016-fx-02).

\section{Author contributions}

B.X.G. and Y.Y.S. conceived and designed the experiments. R.J.Z., Z.Q.L., B.X.G., H.P.L. and S.L.S. reviewed the literature on TB and drafted the manuscript. Y.S., R.J.Z., Z.L., B.X.G., X.F.X., and H.P.L. revised the manuscript. S.L.S. and F.S.H. performed the GWAS SNP chip genotyping. Z.Q.L., J.H.C., J.Z., B.Y.C., Y.H.L., and J.Q.W. performed genotyping of the replication and data analysis. J.Y.C., R.J.Z., Z.Q.L., F.S.H., B.X.G., and Y.Y.S. conducted data analyses. C.H., X.Y.W., H.Y.C., F.L., H.X.W., F.F.X., X.C.C., and X.C.H. recruited samples. J.Y.C., F.L.Z., and S.R.G. generated knockout mice. Y.H.F., L.H.Q., H.Y., Z.H.L., Z.L.C., C.J.Y., J.W., J.H.W., and F.L. performed or contributed to the experiments. All authors critically reviewed and approved the manuscript.

\section{Additional information}

Supplementary Information accompanies this paper at https://doi.org/10.1038/s41467018-06539-w.

Competing interests: The authors declare no competing interests.

Reprints and permission information is available online at http://npg.nature.com/ reprintsandpermissions/

Publisher's note: Springer Nature remains neutral with regard to jurisdictional claims in published maps and institutional affiliations.

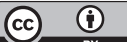

Open Access This article is licensed under a Creative Commons Attribution 4.0 International License, which permits use, sharing, adaptation, distribution and reproduction in any medium or format, as long as you give appropriate credit to the original author(s) and the source, provide a link to the Creative Commons license, and indicate if changes were made. The images or other third party material in this article are included in the article's Creative Commons license, unless indicated otherwise in a credit line to the material. If material is not included in the article's Creative Commons license and your intended use is not permitted by statutory regulation or exceeds the permitted use, you will need to obtain permission directly from the copyright holder. To view a copy of this license, visit http://creativecommons.org/ licenses/by/4.0/.

(C) The Author(s) 2018 